\title{
Algunas consideraciones sobre diccionarios bilingües españoles
}

\author{
Antonio Sánchez Merino
}

\section{INTRODUCCIÓN}

Teniendo en cuenta el auge que se ha producido en la enseñanza de idiomas en España en los últimos años, se ha considerado conveniente analizar el estado de la cuestión en una de las herramientas más importante para dicho aprendizaje, los diccionarios bilingües de español en relación con algunas de las lenguas de su entorno. También se hacen algunos apuntes e indicaciones que pueden contribuir a su mejora en sucesivas ediciones. Para el presente estudio, se analizaron catorce diccionarios que debían cumplir los siguientes requisitos:

(a) debían incluir lenguas con las que el español tuviese una estrecha relación, tanto cultural como geográficamente. Dichas lenguas son el inglés, francés, alemán, italiano y portugués;

(b) debían ser diccionarios de gran tamaño, cada uno debía contener al menos 60.000 entradas en total;

(c) para un par de lenguas dadas no podía haber dos diccionarios de una misma editorial. En esos casos, los diccionarios de menor tamaño suelen ser versiones de los de mayor tamaño.

\section{USOS Y USUARIOS}

Sus posibles usuarios y el uso o los usos para los que esté pensado son los aspectos que determinan de forma general tanto la estructura como el contenido de los diccionarios bilingües, y por eso deben estar claramente enunciados. Sin embargo, ninguno hace indicaciones al 
respecto. Del metalenguaje y de la estructura de los artículos de cada uno de ellos parece deducirse que todos pretenden ser bidireccionales y bifuncionales. Todos pretenden servir para la comprensión y la producción, y cubrir todas las posibles necesidades de todos los usuarios de ambas lenguas. De hecho, son todavía pocos los diccionarios bilingües pensados exclusivamente para los hablantes de español. Se trata de diccionarios concebidos para estudiantes de idiomas, especialmente de inglés, de dimensiones más reducidas que aquellos que han sido estudiados.

Un claro ejemplo de ese deseo de tener utilidad universal lo constituye el $O S D$. Ha seleccionado como variante de pronunciación estándar el inglés americano, cuando por proximidad geográfica parecería más lógico, para un diccionario dirigido a usuarios españoles, optar por el inglés británico. Además, al presentar los equivalentes de las palabras inglesas trata exhaustivamente las variantes geográficas de Latinoamérica, hasta el punto de pasar los equivalentes del español de Europa a un segundo plano. Se prima más la realización de un diccionario dirigido a un mercado lo más extenso posible que la mayor utilidad y calidad que podría tener uno cuyo ámbito de difusión fuese más restringido.

$\mathrm{Al}$ intentar cubrir todas las necesidades de todos los usuarios se descuidan, por fuerza, muchos aspectos, lo que hace que sean deficitarios, ya que las limitaciones de espacio no permiten dar el tratamiento adecuado a cuestiones que sí lo merecen. También puede suponer que información importante, que podría haberse proporcionado si se hubiese hecho un uso más racional del espacio, no se presenta de forma adecuada.

Los diccionarios monofuncionales, para ser usados sólo por los hablantes de una lengua, surgen cuando hay un mercado editorial suficientemente importante, que permite su rentabilización. En el caso del español esto es posible porque es una lengua de difusión mundial, que cuenta con un número creciente de hablantes y con un mercado potencial suficientemente importante como para merecer el esfuerzo editorial que se pueda derivar de ello. La elaboración de diccionarios pensados específicamente para hablantes de español permite a los 
lexicógrafos concentrarse más en cubrir sus necesidades y demandas, en lugar de dispersar sus esfuerzos en dos lenguas. No deja de ser llamativo que sean otras lenguas, con las que el español tiene una relación menos profunda, las que cuentan con diccionarios bilingües monofuncionales. Es el caso del Van Dale Handwoordenboek Spaans Neederlands Neederlands Spaans pensado para neerlandófonos, si bien no excluye su utilidad para hispanohablantes, y del Diccionario español de la lengua china, dirigido a españoles.

La lengua es una de las manifestaciones culturales de la sociedad, que refleja la manera en que sus hablantes ven el mundo. En la medida en que el diccionario bilingüe relaciona culturas diferentes, es necesario que tengan un componente enciclopédico para explicar los aspectos idiosincráticos que no tienen reflejo en la otra lengua. Esa especificidad es independiente de la proximidad geográfica o del parentesco que haya entre ellas, aunque se acentúa en los diccionarios que relacionan lenguas alejadas geográfica y culturalmente, que requieren un mayor componente enciclopédico. Todos los diccionarios estudiados, en la medida en que son diccionarios bilingües, tienen un componente enciclopédico. No obstante, las peculiaridades y la idiosincrasia no siempre se explican adecuadamente, dándose el caso de que muchas veces se produce el establecimiento de relaciones de equivalencia entre palabras que designan realidades no equiparables. Cuando sí se reconoce esa peculiaridad, y el lema va seguido de la correspondiente explicación, no siempre se manifiesta tipográficamente, pudiendo inducir a los usuarios a confusión si no son capaces de discriminar las particularidades del lema. En última instancia, no es extraño encontrar información enciclopédica más propia de un diccionario monolingüe que de uno bilingüe, como hace el $L F E$, al intercalar entre ambos lados del diccionario mapas políticos presentados en láminas en color en solo un idioma.

\section{LAS ENTRADAS}

Las entradas están ordenadas alfabéticamente, y sólo el $D E B$ sigue considerando las combinaciones $\boldsymbol{c h}$ y $\boldsymbol{U}$ como letras individuales. Cuando un mismo lema admite más de una realización ortográfica, y se ha 
considerado conveniente que se recojan, la información puede presentarse de varias formas. Lo habitual es incluir como lema la variante de la realización más extendida o más próxima geográficamente al usuario. En menor medida se usan otras opciones: referencias cruzadas entre las distintas realizaciones en la macroestructura, en entradas no consecutivas; se colocan al comienzo del artículo si son consecutivas, resaltándolas ambas en igual medida; dentro de la microestructura de la variante considerada más importante. La remisión de una entrada a la inmediatamente anterior o posterior como hacen algunos (DSI, SAE, $D P E$ y $D E B$ ) no parece justificada, porque un lugar independiente en la macroestructura sólo tiene sentido en entradas no consecutivas. Una vez decidida la inclusión de dos o más variantes, hay que descartar la duplicación de información, es decir, que se recoja la misma información en entradas diferentes. El EPE duplica la información en todas las entradas a pesar de las referencias cruzadas, lo que no deja de parecer excesivo. Algunos indican entre paréntesis las letras del lema que pueden variar de una forma a otra. La utilidad de recoger variantes ortográficas en los equivalentes depende de que se expliquen las diferencias que puede haber entre uno y otro. Lo lógico, si hay variantes de diferente distribución geográfica, es conceder más importancia a la que está más próxima al usuario. Si ambas formas coinciden en un mismo entorno geográfico, dicha circunstancia ha de indicarse expresamente en la introducción, algo que no hace ninguno de los diccionarios.

Los afijos no son objeto de un tratamiento consistente, ni en lo relativo a su misma inclusión ni en cuanto a la información que proporciona el artículo correspondiente. En la introducción, el $L E I$ dice expresamente que no se encuentran en la macroestructura, pero, contra lo enunciado, luego sí aparecen. En los distintos diccionarios se dan todas las posibles combinaciones de aparición dentro de la macroestructura: se tratan tanto los prefijos como los sufijos (OSD y $C E I$ ), sólo los prefijos (SFE, CDF, DSI, DLEA, SAE y DPE), sólo los sufijos (SAI) o, finalmente, ninguno de ellos ( $L E I, L F E$, Klett, $D E B$ y EPE). La información en cada artículo oscila entre el tratamiento exhaustivo, con información detallada, o la mera indicación del equivalente correspondiente. Se echan de menos ejemplos de uso que muestren contextos de 
empleo en el discurso, salvo en el $D P E$. Esa falta de sistemática se aprecia también en los derivados regulares, que aunque prescindibles, pueden aparecer en entradas independientes. Casi todos reconocen que no merecen un lugar independiente aunque luego se encuentren en la macroestructura (SFE, SAI, DLEA, SAE, DPE, DEB, y EPE), algo menos problemático que indicarlos dentro de la correspondiente a la forma canónica $(D L E A)$.

Las palabras compuestas se suelen encontrar en las entradas de sus elementos integrantes o en la de su primer componente. La información oscila entre la indicación del equivalente, su inclusión dentro de la entrada, como una construcción más, o al final de ésta. En el último caso se procede a su tratamiento en profundidad como corresponde a cualquier otra entrada del diccionario, sin tener en cuenta el elemento del que proceden. No siempre queda claro que se trata de compuestos que juntos pueden tener un significado diferente del que se obtiene con la suma del de sus partes integrantes.

Por cuestiones de espacio y de operatividad, los homónimos deben aparecer siempre en una misma entrada, claramente separados e introducidos por códigos alfabéticos o numéricos, fácilmente identificables, tomando sus diferentes sentidos como principio estructurador del artículo. La problemática en torno a la ubicación de sus equivalentes, en uno o varios artículos consecutivos, es más artificial que real. La utilización de una única entrada para las palabras policategoriales, como se hace con los homónimos, plantea las limitaciones impuestas por la propia estructura de las lenguas. En inglés, algunas palabras pueden tener dos categorías gramaticales dependiendo de cuál sea la sílaba acentuada, y en alemán, una mayúscula o una minúscula inicial puede determinar cambios en la morfología. Incluirlas en un único artículo puede ahorrar espacio, pero lo conveniente es su distribución en entradas diferentes, ya que su pertenencia a varias categorías gramaticales puede pasar desapercibida entre toda la información. Sí puede tener sentido cuando los equivalentes de ambas categorías en L2 sean los mismos, tal y como hacen el $O S D, L E I, L F E, C D F, D S I, D L E A$ y el EPE. Pero en ese caso es de gran importancia que se distingan las diferentes categorías gramaticales. Para que eso suceda, es necesario recurrir a mecanis- 
mos de presentación que eviten que esa circunstancia pase desapercibida. En caso de que los equivalentes difieran dependiendo de la categoría gramatical, lo más apropiado es el uso de entradas sucesivas. Cada una puede ir precedida o seguida de un número volado que permita darse cuenta de que a una misma grafía le puede corresponder más de una categoría gramatical, lo que el $O S D$ hace sistemáticamente, y el $E P E$ ocasionalmente. El empleo de signos tales como guiones o símbolos seguidos de la correspondiente abreviatura gramatical ( $L E I, S F E$, $L F E, S A I$ y $D E B$ ) o de un sistema de numeración (Klett) en una misma entrada puede no ser suficientemente claro. Por otra parte, su auténtico valor como separador de secciones diferentes puede no ser entendido por los usuarios. En un intento de ahorrar espacio, el SAI llega al extremo de presentar lemas a los que corresponden entradas que son claramente diferentes dentro de un mismo artículo.

Las formas irregulares más comunes de cada lengua también son tratadas de diferente forma. Pueden encontrarse en un lugar independiente en la macroestructura, con referencia cruzada al paradigma (OSD, CEI, LEI, LFE, Klett, DLEA, SAI, DPE, DEB y EPE). Otra posibilidad, frecuente en el caso de los verbos, es recogerlas dentro del artículo de la forma canónica (DSI y DLEA). En el caso de las desinencias que se añaden para la formación de derivativos regulares, si se quieren indicar, es mejor colocarlas al final del artículo correspondiente (DLEA).

La falta de información sobre los criterios seguidos para la identificación y el tratamiento en la microestructura de las unidades fraseológicas es generalizada. Sólo se deben incluir en el artículo de uno de sus elementos léxicos, con indicaciones claras al respecto en la introducción. De otro modo, su localización dependerá en gran medida de la buena voluntad y del empeño del usuario. Apenas hacen referencia al tema en las introducciones, excepto el OSD y el DLEA, pero se puede establecer, con carácter general, que para su ubicación en la microestructura, se ha seguido el siguiente orden: primero los sustantivos, después los verbos, y en su defecto, los adjetivos y finalmente los adverbios. No son, salvo en algunos casos, objeto de una identificación clara, por lo que en muchos casos se las confunde con los ejemplos de 
uso del lema. Alguno identifica de forma genérica los enunciados fraseológicos (Klett), sin distinguir entre sus diferentes tipos, mientras que otros, para diferenciarlos de las restantes partes de la microestructura, emplean mecanismos que no son autoexplicativos, tales como barras o guiones ( $L E I$ y $C D F$ ) o signos de puntuación $(S F E)$, entrando alguno en contradicción con lo manifestado en la propia introducción ( $L F E)$. Además, su ubicación dentro de cada artículo individual no sigue una sistemática clara, siendo necesario que tenga un lugar diferenciado en la microestructura que permita identificarlo, como se hace en el $D S /$ y el $D P E$. Su distribución en cada artículo es aleatoria, salvo en el $O S D$ y el DLEA. Es fundamental que se las identifique y utilizar una terminología estándar, fácilmente comprensible por parte del usuario, pues una terminología complicada puede hacerlos inútil (SFE).

Las unidades fraseológicas deben ser objeto de tratamiento diferente dependiendo de su tipo. Por su propia naturaleza, los enunciados sólo tienen cabida en diccionarios de gran tamaño. Para que su inclusión en los anexos de cada lado del diccionario, como hacen el $L F E$ y el $S F E$, sea realmente útil es conveniente que se hagan referencias cruzadas desde la microestructura; si no desde todas las palabras léxicas, debe hacerse al menos desde las más importantes. Para evitar tener que hacer varios índices distintos, lo más práctico puede ser remitir directamente desde la microestructura a las unidades ordenadas numéricamente. Otras cuestiones importantes que se deben hacer explícitas son la posibilidad de que lo que parece ser un enunciado fraseológico equivalente en L2 no sea tal, sino una mera traducción, o de que, aunque lo sean, no puedan emplearse en los mismos contextos, lo que restringe en gran medida sus posibilidades de uso.

Algo más fácil se plante al tratamiento de las construcciones idiosincráticas que si pertenecen al artículo de una de sus partes integrantes. Pero se mantiene el problema de su ubicación, que comparte con las colocaciones. Ni siquiera una cuestión que, en principio, parece tan pacífica como es la inclusión de éstas últimas en la entrada de la base es siempre objeto de un tratamiento adecuado. Contra lo que puede parecer lógico, en algunos diccionarios aparece en el artículo del colocativo ( $L E I, S F E, L F E$ y $S A E$ ), donde muy posiblemente pase inadvertida. 
También debe hacerse explícita su condición como tales de alguna forma, que al mismo tiempo sirve para diferenciarlos de las restantes unidades fraseológicas, ya sea mediante el recurso a tipos de fuente distintos, ya sea con su separación de forma clara dentro de cada uno de los artículos.

\section{LA PRONUNCIACIÓN}

El tipo de usuarios determina no sólo la conveniencia de incluirla o no, sino también el tipo de transcripción que se debe seleccionar. Recoger la pronunciación de las dos lenguas es una muestra más de que el diccionario está pensado para los hablantes de ambas, y de que pretende ser bidireccional. La forma en que se trata adolece de una importante falta de coherencia. La norma es que, en la microestructura, se sitúen detrás del lema, si bien algunos hacen precisiones al respecto al final del artículo ( $S F E$ y $L F E$ ). Hay casos donde, a pesar de que sigue inmediatamente al lema en el artículo, no se trata en la introducción (DSI, $D P E$ y $S A E$ ). Uno incluso prescinde de las tablas que relacionan los sonidos con su correspondiente transcripción (Klett). No siempre cuenta con una sección propia, pudiendo formar parte de la sección dedicada a la gramática ( $S F E$ ) o al alfabeto $(S A I)$. Algunos circunscriben el tratamiento a la Introducción ( $S A E$ y EPE). Las explicaciones en la introducción se presentan siempre en L2, siguiendo la lógica de que son ellos quienes las necesitan. Normalmente, se trata de indicaciones muy generales, que son todo lo que el usuario va a encontrar. No todos tratan con el mismo detalle las dos lenguas, pudiendo primar el tratamiento de una sobre la otra. El $L F E$ se centra casi exclusivamente en el francés, en tanto que el $D E B$ lo hace con el español. Los menos proporcionan un estudio detallado de los sonidos explicando exhaustivamente el sistema de transcripción elegida. Los dividen en consonantes, vocales y semivocales (OSD, CEI, LEI, SFE y DLEA para el alemán), así como también distinguen los diferentes tipos de acentos, primario y secundarios, ortográfico y prosódico (OSD y $S F E)$. Sólo los diccionarios español-inglés diferencian vocales y diptongos.

Las variantes geográficas son objeto de tratamiento en la introduc- 
ción y en la microestructura de los diccionarios bilingües español-inglés en tanto que la divergencia que haya entre ellas pueda ser motivo de confusión. El $O S D$ se centra en las diferencias entre el inglés británico y el estadounidense, y el $C E I$ entre el español de Europa y el de América. En la microestructura, el $O S D$ toma como referente la variante estadounidense, mientras el $C E I$ y el $L E I$ seleccionan como forma de referencia el inglés británico, habitualmente seguido de la variante geográfica, a la que acompaña la correspondiente etiqueta diatópica. Las variantes que no estén relacionadas con la distribución geográfica no están identificadas. Esta circunstancia puede inducir a confusión, puesto que no siempre queda claro que se trate de variantes geográficas. También habría sido de desear que se hubiesen hecho explícitos los criterios que se han seguido para seleccionar las variantes de pronunciación que aparecen, geográficas y sociales, en un apartado dedicado al tema en la introducción, aspecto éste que incluso los diccionarios bilingües español-inglés han descuidado.

El sistema de transcripción elegido no es una cuestión menor. No sirve de nada indicar la pronunciación si para hacerlo se va a usar un sistema con el que los usuarios no están familiarizados y no van a ser capaces de descifrar. La explicación se debe hacer de forma clara, sin ser demasiado compleja. Los sistemas elegidos para representarla se dividen en dos. La mayoría ha preferido el Alfabeto Fonético Internacional o lo han tomado como referente. El $S F E$ y el $D E B$ han creado, exprofeso para el diccionario en cuestión, un sistema que pretende ser autoexplicativo, a partir del alfabeto latino. No obstante, el deseo de proporcionar un sistema claro de transcripción no siempre se consigue con un sistema propio. El $D E B$ compara los sonidos de otros idiomas para aclarar el modo en que se pronuncian el español y el portugués. Por otra parte, no hay que olvidar que muchos usuarios de diccionarios bilingües de gran tamaño son, o bien estudiantes avanzados del idioma o bien profesionales con conocimientos de fonética. Tener que aprender un sistema específico de una sola obra obliga a una dispersión de esfuerzos que los usuarios pueden no estar dispuestos a hacer. La necesidad de explicar todas las peculiaridades de la pronunciación con un sistema propio de transcripción supone que debe haber una sección en la 
introducción dedicada a ella. También es muy desafortunada la decisión del $C D F$ de indicar sistemáticamente el acento prosódico gráficamente sobre la forma canónica de las palabras italianas, cuando no se emplea en dicha lengua mas que en casos muy contados, puesto que se proporciona información de forma tal que puede inducir directamente al error.

Con frecuencia, la pronunciación de una palabra, o de alguno de sus sonidos, varía entre la forma aislada, que es la que se recoge de forma sistemática en todas las entradas de todos los diccionarios, y su producción en el discurso hablado. Esa peculiaridad se muestra, en los diccionarios que la indican, de varias formas: escribiendo el sonido afectado entre paréntesis $(O S D)$, en cursiva (CEIy $D S T)$, con una tipografía diferente (CEIy Klett), o utilizando signos (LEI).

La amplitud de la información en cada entrada oscila entre el tratamiento completo de algunos diccionarios de ambos lados del diccionario ( $D S I$ y $E P E)$, de sólo uno de ellos en los de español-inglés, y su completa ausencia en ambos lados $(D P E)$. Aprovechando que no suelen plantear demasiados problemas a los hablantes extranjeros, la pronunciación de las palabras españolas se recoge de forma bastante limitada, salvo contadas excepciones, referida a sonidos que se consideran problemáticos (LEIy Klett), sin explicar en ningún caso a qué se hace referencia con dicha problemática, a acrónimos $(O S D)$, o a algunos sonidos de algunas palabras $(D E B)$. El tratamiento de la pronunciación de la otra lengua es más detallado, oscilando entre el tratamiento exhaustivo y la inclusión sólo de sonidos aislados que se crea puedan ser problemáticos, criterios tampoco explicados. La mayoría, si bien no recoge toda la pronunciación de uno de los dos lados de forma exhaustiva, sí trata la de muchas de sus entradas, sin que se expliquen los criterios que se han seguido para decidir reproducir la pronunciación de unas y no de otras. No es extraño encontrarnos con diccionarios que aprovechan la parte común de la pronunciación de una palabra al indicar la siguiente, para señalar sólo lo que es diferencial, sustituyendo la parte coincidente por un guión ( $L E I$ y $L F E$ ). También es posible encontrarse con que sólo se reproduce la pronunciación del comienzo de la palabra (Klett) o sonidos aislados (DLEA). Una vez que se ha decidido incluir- 
la, debe indicarse completa en todas las entradas del diccionario. Recoger sólo la pronunciación de determinados fonemas o palabras no conlleva el ahorro de un espacio suficientemente significativo como para merecerlo, y puede acabar por desorientar a los usuarios. El OSD, el $L E I$ y el $L F E$ tratan la pronunciación de formas distintas a la canónica pero, a pesar de ello, tampoco de forma exhaustiva. Poca atención merece la pronunciación de las formas compuestas que tienen entradas independientes en la macroestructura; de hecho, únicamente se tratan en el $C E I$.

\section{EL ASPECTO GRAMATICAL}

Las cuestiones gramaticales se tratan en la microestructura y, en muchos, también en otras partes del diccionario. Se puede encontrar indistintamente en la introducción o en anexos o apéndices al final de uno o de los dos lados del diccionario. Esas secciones son de calidad y extensión muy variada, llegando, en alguno de ellos, a asimilarse a cualquier gramática al uso, con secciones dedicadas sucesivamente a las distintas partes de la oración. Esas gramáticas son especialmente exhaustivas en los diccionarios de francés. Se abusa de la información relacionada con cuestiones morfológicas, en detrimento de la información sintáctica, que apenas se recoge pero que, a pesar de ello, es igualmente importante. Sólo el $O S D$ ofrece, en la sección correspondiente, información sobre construcciones sintácticas modelo.

Al tratarse de diccionarios de gran tamaño, es de esperar que el usuario tendrá a su alcance y usará gramáticas de las lenguas correspondientes, a las que acudirá para realizar las consultas oportunas. Esto no quiere decir que la información que los diccionarios proporcionen al respecto sea redundante. Pero, sin lugar a dudas, será de mucha más utilidad cuando exista una relación directa entre la que aparece allí y la que se proporciona en la microestructura, como hacen el $O S D$, el CEIy el Klett. En este sentido, son especialmente útiles las referencias cruzadas que, desde los artículos de todos o algunos verbos, se hacen a anexos en los que se detallan, total o parcialmente, conjugaciones verbales modelo (OSD, CEI, SAI, Klett, DEB y EPE). Por lo demás, las relacio- 
nes acaban ahí. Los anexos se presentan en ambos idiomas total (OSD), o parcialmente (CEI), habiendo sido pensados expresamente siempre para los usuarios de L2 ( $L E I, S F E, L F E$, SAI y $D E B$ ), o sólo para una misma lengua $(S A E)$.

Se echa en falta que no haya referencias a las diferencias morfológicas y sintácticas más importantes que pueda haber entre ambas lenguas, especialmente los aspectos que son exclusivos de cada uno de ellas. La indicación de esas diferencias no tienen que estar dentro de los artículos, porque su extensión puede hacer excesivamente complejo el manejo del diccionario, o porque se exceda de la información que corresponde al artículo, pero sí debe hallarse en la introducción o en los apéndices a los que se hagan referencias cruzadas.

Aunque no sean un elemento central dentro del artículo, ni la información que en mayor medida demandan los usuarios, según han demostrado diferentes estudios, los diccionarios bilingües españoles prestan muy poca atención a las cuestiones gramaticales dentro de la microestructura, en contraposición a lo que pueden llegar a a ser farragosos compendios gramaticales de otras partes del diccionario. La gran mayoría se conforma con una simple clasificación con muy diferentes formas de indicación: entre paréntesis, en cursiva, en minúsculas, ... Cuesta entender que muchas veces se limite a una breve caracterización morfológica de la palabra de la entrada y, ocasionalmente, de sus equivalentes, y, al mismo tiempo, se puedan dedicar más de diez páginas a tratar cuestiones gramaticales muy elementales, del tipo de las que se encuentran en una gramática escolar al uso. El contraste es aún más llamativo si se piensa que muchas veces da la impresión de que esas pequeñas gramáticas son superfluas: contienen información tan básica que es prescindible, como pueden ser las listas de números ordinales.

La forma gramatical del lema es la forma canónica en la tradición lexicográfica occidental. De acuerdo con ésta han sido etiquetadas todas las entradas en los distintos diccionarios según el sistema tradicional de clasificación de las partes de la oración: artículos, sustantivos, pronombres, adjetivos, verbos, adverbios, preposiciones, conjunciones e interjecciones. En el caso de los sustantivos, la forma canónica es 
siempre la correspondiente al nominativo masculino singular, salvo en el LEI donde, si la forma femenina es más habitual que la masculina, es aquella la que encabeza el artículo. De acuerdo con la tradición lexicográfica española, los sustantivos españoles no siempre están etiquetados como tales, y varios sustituyen la abreviatura correspondiente al sustantivo por la correspondiente al género, o géneros cuando se diferencia entre dos. Su caracterización como tal, o la indicación de su género, acompaña también a la de su equivalente si no hay coincidencia en el género, salvo en el $D E B$. Puesto que la forma femenina no siempre se deriva regularmente de la masculina, es conveniente que vaya seguida de la desinencia irregular cuando corresponda. Al mismo tiempo sería conveniente que, en la introducción o en las indicaciones de uso, se enunciasen los principios seguidos para recogerla. Por ejemplo, en el caso del español el límite de exclusión es la regla básica de formación del femenino, cambiando la vocal o por la a. Igualmente, cuando una misma forma sirve para ambos géneros, esa peculiaridad se debe hacer explícita mediante las correspondientes abreviaturas. En caso de que se trate de palabras que puedan tener significados no relacionados entre sí, el tratamiento más apropiado depende del uso que se le quiera dar al diccionario. Si se espera que sirva para la producción, es deseable que los dos equivalentes aparezcan uno a continuación del otro. En los de comprensión cada uno debe aparecer en el lugar que le corresponde en la macroestructura. Toda la información se puede encontrar a continuación de la entrada o puede hacerse una referencia cruzada a la otra forma. Ninguno, al margen del DSI y el EBE, donde aparecen tanto para los lemas seleccionados como entradas como para sus equivalentes correspondientes, la ha incluido de forma coherente y consistente en la macroestructura. Si no coinciden el género del lema y el del equivalente esta circunstancia siempre se hace expresa indicando su género a continuación del equivalente.

Casi todo lo que se ha dicho en relación con las formas femeninas se puede aplicar por analogía al tratamiento que se debe dar a los plurales. Se deben especificar todas aquellas particularidades derivadas de la formación del plural, como los singularia y los pluralia tantum con las correspondientes indicaciones. Sin embargo, todos tratan estos aspectos 
de forma bastante inconsistente, algunos de ellos enunciando principios que no siempre siguen. Pueden hacerse explícitos junto a la propia abreviatura ( $D S /$ y $C D F)$, con la desinencia correspondiente si hay alguna particularidad que haga que no se ajuste a la formación del plural, con la forma completa en caso de que no se pueda derivar del singular, o con un signo si dicha forma es invariable para ambos números $(O S D$, $C E I, L E I$ y $L F E$ ). Una última opción, empleada por los diccionarios Larousse es relegarlos, en algunos casos, a una sección que sirve como cajón de sastre bajo el nombre genérico de observaciones ( $L E I$ y $L F E$ ). $\mathrm{Si}$ es acertado en el caso de artículos cortos, pierde validez cuando su extensión hace que dicha información no esté próxima ni al lema ni a su equivalente. La mayoría se limita a hacer indicaciones esporádicas.

En cuanto a una caracterización más profunda de los sustantivos, de todas las posibles divisiones que se pueden establecer, solamente se diferencia en todos los diccionarios estudiados entre nombres comunes y nombres propios, cuando se incluye a éstos últimos. Únicamente el $O S D$ y el $D S I$ diferencian los lemas entre sustantivos contables e incontables. Y, en el caso de que no los identifiquen, no siempre puede el usuario deducir que se trata de unos u otros a partir de la información que proporcionan los ejemplos.

Los pronombres son objeto de una identificación genérica muy vaga, salvo excepciones ( $C E I, L E I$, Klett, DLEA, DEB y EPE), y eso en apenas algunas entradas, en las que se hace referencia tanto a ulteriores subdivisiones como a sus posibles funciones sintácticas. El etiquetado es insuficiente, porque no basta con que se indique que un pronombre determinado es un pronombre personal. Si el usuario sabe identificar correctamente un pronombre, casi con toda seguridad será precisamente un pronombre personal: más problemas tendrá para poder distinguir un pronombre relativo, demostrativo o indefinido. Y, curiosamente, son los pronombres personales los que van acompañados sistemáticamente de las abreviaturas características. Por otra parte, también sería conveniente que se hiciera extensivo a todos los diccionarios el tratamiento de cuestiones sintácticas, ya que es un tema al que casi todos son completamente ajenos. 
La forma seleccionada para los adjetivos es el masculino singular, en su grado positivo, identificados de forma genérica como tales. La forma masculina va seguida, no siempre, ni en todos los diccionarios, de la desinencia correspondiente al femenino. De forma consistente, sólo sucede en el CEI, el LEIy el SFE. Del mismo modo, sólo acompaña al equivalente en algunos casos. En cuanto a posible subdivisiones, los más completos son los diccionarios bilingües español-inglés, pero no exhaustivamente. El $O S D$ y el $C E I$ diferencian a veces entre adjetivos predicativos y atributivos. El tratamiento que se debe dar a la formación de los comparativos que no se hagan por derivación, así como a las cuestiones referentes a su número y género, ha de ser el mismo que el que se da a los sustantivos. Si las formas comparativas y superlativas no pueden derivarse de la forma positiva se debería elegir entre incluirla completa tras el paradigma, o en su lugar correspondiente en la macroestructura, dependiendo de si se pretende que se haga un uso productivo o comprensivo del diccionario. Por supuesto, si se pretende que sirva para ambos usos, deberán recogerse en ambos lugares, aunque ello suponga la duplicación de determinada información, algo a lo que los diccionarios no deben ser ajenos. Han resuelto la cuestión de diferentes maneras, ninguna completamente satisfactoria desde el punto de vista del usuario: pueden aparecer dentro de la microestructura de la forma positiva (Klett y $E P E$ ), en su lugar correspondiente en la macroestructura (CEI, DSI, DLEA, SAE, DPE, DEB y DEB), con referencia a la forma positiva o sin ella, y, en última instancia, no aparecer en ningún caso ( $L F E)$.

Todos recogen proporcionalmente mucha más información en las entradas correspondientes a los verbos de la que contienen al tratar las restantes partes del discurso. Ni siquiera en este caso es satisfactorio el tratamiento gramatical de las entradas, más necesario que en el de las restantes partes del discurso por las implicaciones sintácticas y semánticas que se derivan de sus características intrínsecas. Ninguno es exhaustivo en su clasificación verbal, lo cual no es obstáculo para que el $C D F$ cree una categoría nueva, la de los verbos despectivos ( sic), confiriéndole el mismo valor que a otras categorías establecidas y aceptadas. Es el caso, por ejemplo, de los verbos defectivos, por mencionar sólo una 
categoría que ningún diccionario recoge. Esta falta de precisión es más seria de lo que parece, porque dos diccionarios llegan a emplear una misma abreviatura, y por tanto, considerarlos equivalentes a verbos pronominales y reflexivos $(D S I)$ o a verbos reflexivos y recíprocos $(C E I)$. La lingüística es una disciplina que está en constante evolución y es conveniente que, en la medida de lo posible, gramáticas y diccionarios compartan una misma terminología. Lógicamente, no se puede esperar que los diccionarios reflejen de forma sistemática las tendencias más recientes e innovadoras, pero sí sería de agradecer que al menos delimitasen de forma clara cuestiones que tienen tanta trascendencia como las enunciadas. Al margen del infinitivo también algunos presentan en la macroestructura otras formas no personales, participios de pasado y de presente ( $L F E, D P E$ y $D E B$ ). Algunas formas irregulares ocupan un lugar propio en la macroestructura de algunos diccionarios, desde donde se remite a la forma canónica ( $S F E, S A I, D P E$ y $E B E$ ) mientras otros la incluyen directamente en la entrada de aquella, a continuación del lema (OSD, SAI y CEI) o al final de artículo (LEIy Klett).

La forma canónica de los verbos es el infinitivo activo simple. Es la forma a partir de la cual los usuarios pueden derivar con más facilidad las restantes formas del paradigma. Algunos diccionarios cuentan con distintos mecanismos para ayudar a que los usuarios deriven las restantes formas del paradigma. El CEI hace una remisión mediante código alfanumérico a un anexo que incluye conjugaciones tipo, donde recogen, bien todos los tiempos verbales, bien los más importantes tanto de verbos regulares como irregulares. Hay otros más simples pero menos útiles, como el $L E I$, con indicación de la irregularidad mediante asteriscos o indicación del verbo a cuya conjugación se asemejan; y, en ocasiones, incompletos por la escasez de información que proporcionan, inclusión a continuación de la forma del paradigma de los cambios que se producen en la raíz (Klett). El inconveniente derivado de la utilización de alguno de esos mecanismos es que el diccionario lo pueda aprovechar para cuestiones que no tienen nada que ver con dicha irregularidad $(L F E)$. No todos los diccionarios hacen indicaciones sobre la irregularidad del paradigma; no lo hacen el $D S I, C D F, S A I$, Klett, $D P E$ ni el $E B E$. 
En el caso de los adverbios y las preposiciones, el tratamiento ha sido, en gran medida, el mismo que el de los pronombres: una simple etiqueta de carácter general sin mayores precisiones. Sólo el Klett va algo más allá al indicar algunas particularidades sintácticas relacionadas con el régimen de las preposiciones en alemán. Esta opción es especialmente insatisfactoria, por insuficiente, en el caso de los adverbios, que en ningún diccionario y para ningún idioma, aparecen clasificados de acuerdo a su significado (de tiempo, de lugar, de cantidad, ...). La abreviatura también es genérica para las conjunciones, con alguna información sobre restricciones de uso (CEI y $L F E$ ) e indicaciones sobre su subclasificación (DPE y $D E B)$.

La información sintáctica en la microestructura, sin llegar a tener gran importancia, está más desarrollada, como sucede con la pronunciación, en los diccionarios español-inglés y en el DSI. En otros se trata de manera muy puntual ( $S F E$, Klett, $D E B$ y $E P E$ ), sin que sea extraño que recaiga en el propio usuario la responsabilidad de deducirla a partir de los ejemplos $(L F E)$. La necesidad de desarrollarla se da en mayor medida si se pretende que se pueda usar el diccionario para la producción. Aún así, en el mejor de los casos, se limitan a indicar restricciones de colocación y concordancias, régimen de construcción de determinados verbos, así como algunas valencias, que no siempre aparecen identificadas como tales, sino disfrazadas de ejemplos. Convenientemente, para la presentación de la información sintáctica, cuando lo han hecho, los diccionarios se han valido de ellos, códigos autoexplicativos muy simples. Se echa de menos que cada construcción no vaya seguida no sólo de una equivalente, sino también de un ejemplo que clarificase los usos, lo que se puede explicar porque se espera que dicha construcción sirva a la vez como ejemplo.

\section{LOS EQUIVALENTES}

Constituyen el elemento central y más elaborado de todos los diccionarios, si bien hay aspectos de su tratamiento que son mejorables. Es más difícil de lo que parece encontrar palabras que sean equivalentes perfectos entre dos lenguas. Y, en consecuencia, es imposible emparejar 
todas las palabras de dos lenguas. Por la misma razón, aún será más difícil encontrar dos o más equivalentes, porque éstos deben ser, a su vez, equivalentes perfectos entre sí. A pesar de esto, parece deducirse de las entradas de algunos de los diccionarios analizados que la equivalencia perfecta no sólo no es rara sino que incluso es la norma. En ellos se listan indiscriminadamente equivalentes de L2 separados por signos de puntuación, comas los más de los casos, $\mathrm{y}$, menos habitualmente, punto y coma. Es precisamente esa forma de separación la que induce a confusión. Nos encontramos así con que una cuestión que, en principio, es tan pacífica como la separación de los distintos equivalentes dentro de la microestructura, no se resuelve siempre de la forma más apropiada.

Si sólo hay un equivalente para el término de la entrada, y no hay ninguna peculiaridad en el significado de ninguno que restrinja las posibilidades de intercambio, lo habitual es que el artículo concluya con ese equivalente, al considerarse los ejemplos como meramente contingentes. No obstante, en el DPE a cada equivalente le acompaña sistemáticamente una definición del estilo de las que se encuentra en un diccionario monolingüe, a las que el $S A E$ recurre más esporádicamente y el $S F E, L F E, C D F, D L E A$ y el $D E B$ ) muy de cuando en cuando. Su longitud es muy variable, $\mathrm{y}$, sirve para calificar a ambos, sin que quede demasiado clara su utilidad.

Un gran número de palabras en cada lengua sólo tienen equivalentes parciales en otras, que no cubren por sí solos todos los matices de la palabra de L1. Sin embargo, con frecuencia se abusa del mencionado listado indiscriminado de supuestos equivalentes uno detrás de otro, separados simplemente por comas, que no va hacer que el diccionario sea más útil. Incluso concediendo que el valor de esos supuestos equivalentes no sea tal, y que se hubiesen incluido con la finalidad de servir como mecanismo de discriminación (parónimos, sinónimos ...) más que como auténticos equivalentes, la forma en la que se presentan no hace esa circunstancia evidente. Sólo un usuario cualificado podría aprovechar esa forma de presentación y sacarle todo el partido.

Si no se puede establecer esa relación de equivalencia perfecta, es necesario que entren en juego los mecanismos de discriminación de 
significado. Tienen como misión indicar, de todos los contextos de uso del término de la entrada, en cuál o cuáles se puede emplear el equivalente correspondiente. Su presencia debe ser, en todo caso, independiente de que para el lema haya uno o más equivalentes parciales. Sin embargo, muchos diccionarios los usan de manera bastante inconsistente, no indican los matices de ninguno de los equivalentes, y cuando lo hacen, no los utilizan con la profusión con la que sería deseable. Si ya es de por sí delicado cuando la equivalencia entre dos palabras es parcial, mucho más complicado es aún cuando al lema le siguen varios equivalentes. Muchas veces, los usuarios, sobre todo los menos cualificados, se suelen conformar con el primero o, a lo sumo, con el segundo de los equivalentes que se le presentan, sin verificar su utilidad. Cuando las palabras sólo son parcialmente equivalentes no se debe forzar la situación para emparejar unidades léxicas como si fuesen absolutamente equivalentes. Un sistema que está bastante extendido, pero cuyas posibilidades no se han aprovechado suficientemente dentro de cada obra individual, es la posibilidad de la indicación de contextos y palabras de L1 con las que se puede usar precediendo a los equivalentes correspondientes (OSD, LEI, SFE, LFE, DSI, CDF, SAI, Klett, DLEA, SAE y $D P E)$. Pueden usarse también para discriminar a los equivalentes ( $L E I$, SFE y $S A I)$.

No obstante, no se trata únicamente de que, cuando haya más de uno, cada equivalente parcial vaya acompañado de los pertinentes mecanismos de discriminación de significado. Deben estar a su vez claramente separados en el artículo. El recurso, como ya se ha dicho, a signos de puntuación, induce a pensar que son sinónimos entre sí. La incertidumbre aumenta cuando, a lo largo del diccionario, en diferentes entradas se emplean distintas formas de separar equivalentes $(C D F)$. Si se trata de varios equivalentes que no son sinónimos entre sí, la mejor manera de evitar malentendidos es que se presenten separados numérica o alfabéticamente (OSD, CEI, DSIy Klett). Para hacer aún más evidente la particularidad que permite al usuario saber más claramente dónde encontrar el equivalente que está buscando, lo más práctico es que cada uno de los bloques vaya precedido por un sinónimo o un parónimo, lo que sólo hace el CEI. Otra opción, separarlos tipográficamen- 
te mediante barras verticales está bastante extendida. Su utilidad se ve, no obstante, limitada en los diccionarios donde este sistema coexiste con la separación mediante signos de puntuación ( $L E I, L F E, C D F$ y $D L E A$ ). Tampoco contribuye especialmente a facilitar el manejo del diccionario que alguno aproveche, para separar diferentes equivalentes no relacionados, los mismos signos de puntuación que se usarían si los equivalentes fuese intercambiables ( $L E I, S F E, L F E, S A I, S A E$ y $D E B$ ). En el $D S I$ cada equivalente va etiquetado por orden alfabético, a fin de que no haya malentendidos, salvo en los casos en los que se considera que más de un término de L2 es equivalente exacto del original de L1.

Si a su vez los sentidos de cada bloque son susceptibles de sucesivas subdivisiones por tener el lema muchos matices, es aconsejable presentarlos en bloques diferenciados, donde los sentidos se agrupen según su afinidad en L2, lo que solamente hace el $O S D$. Emplea para la subdivisión de palabras en una misma entrada, dependiendo de su complejidad semántica, no sólo la numeración arábiga y la romana sino también la división alfabética. No hace falta decir que cada sección debe ir encabezada a su vez por algún mecanismo de discriminación de significado.

Cuando un equivalente parcial no es suficiente para referir el concepto original, los diccionarios suelen valerse de pequeñas perífrasis que permiten introducir los matices de los que una sola palabra carece. Su brevedad facilita su sustitución en el contexto de L2. La lógica dicta que para que sean útiles se escriban en L2, cosa que hacen todos con excepción del $L E I$, que los presenta en L1. Si no se considera suficiente, o no son suficientemente explícitos equivalentes y perífrasis, se recurre, en última instancia, al empleo de definiciones, también en L2. Una explicación puede ser más útil que un irreal equivalente de traducción, especialmente en el caso de las palabras culturalmente específicas, para las que no es suficiente con una simple perífrasis. Con una breve definición que recoja los matices del término es suficiente para permitir al usuario que la entienda y que, llegado el caso, la use en contextos adecuados. En caso contrario, se puede crear una falsa sensación de relación entre los términos. Es importante que se diferencien tipográficamente de los demás elementos de la microestructura, lo que de forma habitual hacen el $O S D$, el $C E I$ y el Klett. El $L E I$, el $D L E A$ y el 
EPE lo hacen ocasionalmente, y los restantes nunca. Buena prueba de lo necesarias que son algunas definiciones y de la dificultad de explicar esos conceptos en L2 es la necesidad de incluir términos de L1 (OSD y $S F E$ ). De otra manera, deberían presentarse dos definiciones, una dentro de otra, haciendo la explicación demasiado enrevesada para que fuese comprensible.

Los diccionarios estudiados abusan de los equivalentes de traducción, presentando como tales palabras que realmente no lo son, sin utilizar explicaciones, perífrasis o mecanismos de discriminación de significado, que son necesarios en muchos casos, haciendo creer que existe una relación de equivalencia cuando en realidad no son tales. Es cierto que, ocasionalmente, en algunos diccionarios se intenta concienciar al usuario de que dicha relación de equivalencia no existe realmente. Así, los hay que utilizan símbolos matemáticos (OSD, CEIy Klett) o signos ( $L E I$ ) para indicar que el término seleccionado no se trata de un equivalente propiamente dicho. Sería conveniente que esos "equivalentes" fuesen acompañados de mecanismos de discriminación de significado más claros, como hace el Klett, que añade notas explicativas en cursiva entre paréntesis y en L2. Los símbolos sólo aclaran que no existe esa correspondencia perfecta, pero nada más. No indican los contextos en los que el equivalente no se puede usar o los matices que los diferencian, que es precisamente lo que más necesita saber el usuario.

Una posibilidad que no se ha desarrollado suficientemente, y que sería interesante explorar como alternativa, es la de los equivalentes neutros, procedimiento por el cual los elementos de L1, ya sean unidades simples o multicompuestas se descomponen semánticamente, y sus componentes criteriales se traducen a L2. Donde más se ha avanzado en este campo ha sido en los diccionarios español-inglés y en algún que otro más (Klett y $S A I$ ). Es una medida que, para ser más eficaz, también requeriría que fuesen acompañados de explicaciones en L2, de la forma en que se hace en el LEIy en el LFE. Estas son necesarias porque, en tanto que el equivalente puede no estar asentado dentro de la lengua que lo recibe, no aporta datos al usuario sobre su sentido y sus posibilidades de uso. Otras veces han optado, llegando a abusar de ellos, por los calcos semánticos, en los que se repite el mis- 
mo problema: la ausencia de indicaciones, bien sea de discriminaciones de significado, bien sea de definiciones que acoten el sentido del término.

Con diferencia el recurso más empleado en todos los diccionarios, las etiquetas pueden llegar a ser difíciles de entender y aprovechar por el usuario. Un primer problema se deriva de su ubicación en la introducción. Allí se encuentran agrupadas de forma indiscriminada y ordenadas alfabéticamente todas las abreviaturas que se han empleado en el diccionario, sin que no siempre se distingan tipográficamente según su tipo. Este tratamiento indiferenciado transmite la impresión, con independencia del valor que se le otorgue, de que se le concede poca importancia. Más allá de esa forma de presentación, que es la adoptada por la gran mayoría de los diccionarios, todos se mueven entre una explicación exhaustiva de su significado y la ausencia total de referencias al tema en ninguna parte, de forma que ni siquiera se explica, y es el usuario quien debe deducir su significado usando el sentido común. Lo habitual es que no se explique suficientemente. En casi todos simplemente se enuncian. El $O S D$ las explica y separa en bloques según su sentido: diacrónicas, diatextuales, diatópicas, diafásicas y diaevaluativas. El DLEA añade etiquetas diaintegrativas y diatécnicas. Al no explicarse, su sentido y utilidad no queda claro muchas veces. Mucho más delicado es que una misma etiqueta haga referencia a más de un concepto, como sucede en el $L F E$, el $C D F$ y el $D S I$. No es aceptable, con independencia de lo mucho o poco que puedan estar relacionados. Suelen ser las mismas para ambas partes del diccionario. En el $O S D$ se diferencian según sirvan al lema o al equivalente: cuando hacen referencia a la palabra de la entrada preceden a todos los equivalentes, mientras que si sólo son de aplicación al equivalente siguen a éste último. Otros son tan sutiles en su presentación que pasan fácilmente desapercibidas. En el $L E I$ y en el $L F E$ si se refieren al lema se escriben en mayúsculas y preceden a los equivalentes, mientras que van en minúsculas y a continuación de ellos si se refieren a estos últimos. 


\section{LOS EJEMPLOS}

Para indicar el uso en el discurso de los lemas y los equivalentes, se valen de textos en prosa, la mayoría sintagmas o breves oraciones y, de forma más esporádica, pequeños diálogos (CEIy LEI). Son varios los recursos que hay para ahorrar espacios. Los sujetos se sustituyen por formas abreviadas de pronombres indefinidos (LEIy DLEA) que no siempre se explican en la introducción, o se sustituye la parte de la oración que no aporta nada al ejemplo por puntos suspensivos (CEI, Klett y $D L E A$ ). El lema se ha sustituido por una tilde en la medida de lo posible, excepto en el $D S I$, a los que se añaden las correspondientes desinencias ( $O S D$ y $C E I)$. El $C D F$ va más allá y sustituye el equivalente en la traducción del ejemplo por una abreviatura. Los verbos, tanto en forma personal como no personal, también pueden ser sustituidos total o parcialmente, aprovechando en la medida de lo posible el lema. En principio, ambos sistemas parecen igualmente válidos, pero desde el momento en que no se pueda aprovechar el lema en la construcción de ejemplos de formas personales de los verbos, la sustitución parcial parece una solución bastante cuestionable. La necesidad de añadir terminaciones al guión o símbolo que sustituye al lema puede obligar al usuario a tener que decidir hasta qué punto es aprovechable el lema en el ejemplo, lo que no siempre queda claro.

Deben seguir inmediatamente a cada equivalente con los que se empleen, aunque hay quienes los colocan agrupados de forma indiscriminada al final de cada artículo (CEI, LFE y $S A I)$. Salvo el $D P E$, que no traduce los ejemplos españoles al portugués, los demás traducen todos los ejemplos. Son uno de los componente que requiere una puesta al día en todos los idiomas, no sólo por lo obsoleto de las construcciones que se usan, sino también por las importantes faltas de coherencia que se observan. En más de la mitad de los diccionarios se aprecia en los ejemplos una discordancia importante con los equivalentes del lema. Se espera que en la correspondiente traducción aparezca alguno de dichos equivalentes; sin embargo, al efectuarla no se ha usado ninguno de los que aparecen en el artículo (CEI, LEI, SFE, SAI, CDF, SAE, DLEA, Klett y $D P E$ ). Esto sólo puede significar que, o bien no se han cubierto 
todos los sentidos posibles del lema de L1, o bien que el ejemplo no es realmente indicativo de un uso libre en el discurso de dicha palabra. Se trata de un caso que es completamente distinto de aquel en el que el lema está inmediatamente seguido de un ejemplo de su uso en L1 y su correspondiente traducción. Una posible explicación, que no excluye la conveniencia de haber realizado una explicación, es la dificultad de expresar el concepto que engloba el término original en una palabra $(O S D)$. Otras cuestiones que limitan su utilidad son que, en más de un caso, ha sido necesario explicar el ejemplo para que el usuario lo entienda y que, en más de un diccionario, se ha usado un mismo ejemplo para indicar tanto una cosa como la contraria.

Si bien sería muy útil que cada equivalente fuese seguido de un ejemplo de uso, hay que ser consciente de la imposibilidad de realizarlo por las consabidas limitaciones de espacio, pues perfectamente podría significar duplicar el tamaño final de la obra. A pesar de eso, algunos diccionarios dan, para algunos ejemplos, más de una traducción, del mismo modo que también es posible que una misma traducción a L2 le correspondan dos textos en L1 (LEI).

Si se ha optado por agruparlos al final del artículo, no deben entremezclarse con las unidades fraseológicas, ya que si lo hacen pueden confundir a los usuarios (CEIy $L F E$ ). Así, se podrá diferenciar claramente cuál de las construcciones que acompañan a un equivalente es un ejemplo y cuál se corresponde con su uso dentro de una unidad fraseológica. Pero hasta cuando no están agrupadas, no se distinguen claramente unas de otros, y se valen del mismo tipo de presentación, cursiva o negrita, en ambos casos. Distinciones tipográficas, como el cambio del tipo y tamaño de fuente, al modo en que hace el $O S D$, en una obra donde el espacio está tan aprovechado como en un diccionario, puede pasar perfectamente desapercibida. La inclusión de etiquetas o signos identificativos que califiquen a los enunciados fraseológicos o a los ejemplos no se hace con la consistencia necesaria en ningún diccionario analizado, salvo el $D S I$, el $C E I$, el $C D F$ y el $S A I$. El empleo de signos al estilo de los usados para separar equivalentes parciales para diferenciarlos es bastante confuso ( $L E I, S F E, C D F$ y $D L E A$ ), y no siempre se aplican con coherencia $(L F E)$. También es problemático el 
uso como ejemplos de construcciones idiosincráticas o incluso locuciones que no proporcionan al usuario una visión clara sobre el posible empleo de esa palabra en un contexto real, sino que incluso pueden llegar a desconcertarlo en la medida en que refleja, bien al contrario, un uso muy particular.

\section{LAS ILUSTRACIONES}

Las ilustraciones, si bien no son una parte fundamental de los diccionarios bilingües, pueden ser muy prácticas a efectos de la presentación de algunos elementos culturales propios. No obstante, al margen de los pictóricos, muy pocos diccionarios bilingües españoles las usan, salvo los diccionarios para estudiantes, que hacen de ellas un uso eminentemente lúdico. De todos los diccionarios estudiados, sólo se encuentran en el $L E I$, que en las páginas centrales presenta diversas láminas en color, todas las cuales hacen referencia a construcciones humanas (un automóvil, un avión, una máquina de ferrocarril, una casa, ...). Es un recurso al que no le ha sacado todo el partido posible, limitándose a una utilización fácil y más bien efectista. Incluso en él parecen haberse recogido más como curiosidad que por la utilidad que puedan tener.

\section{CONCLUSIONES}

Son varias las conclusiones que se derivan del presente estudio. En cuanto a la situación general, llama la atención la gran diferencia que hay entre la calidad de la lexicografía bilingüe español-ingles y la de las restantes lenguas; los diccionarios bilingües español-inglés son los más elaborados, lo que podría explicarse por el mayor interés existente por esa lengua en nuestro país. Esta puede ser también la razón que justifique la existencia de diccionarios bilingües español-inglés pensados exclusivamente para hablantes de español e incluso de tres diccionarios semibilingües.

Es necesaria una revisión en profundidad de algunos aspectos de la lexicografía bilingüe española, que está por detrás de la de otras lenguas de su entorno que tienen un peso relativo mucho menor, no sólo 
por difusión sino por producción. Específicamente, es urgente que se proceda a aplicar a la práctica lexicográfica bilingüe española los resultados de los estudios teóricos que se han desarrollado en los últimos años. Es especialmente el caso de aspectos no nucleares de este tipo de diccionarios, como son la gramática o la organización de la información en la macroestructura. Asimismo, para poder proceder a una mejora sustancial de su calidad hay que realizar estudios que permitan conocer realmente que es lo que les piden y lo que esperan los usuarios españoles de este tipo de obras.

Todos presentan inconsistencias y falta de sistemática dentro de una misma obra. Es comprensible hasta cierto punto en obras que llegan a superar, en muchos casos, las 200.000 entradas. Pero es difícilmente entendible que enuncien expresamente principios que contradicen en la microestructura. También es cuestionable que, por una parte, se pretenda ahorrar espacio suprimiendo información que no siempre es prescindible (pronunciación de palabras, información gramatical, separación inadecuada de ejemplos y unidades fraseológicas, ...), o no proporcionando la suficiente, cuando sería necesario. Pero por otra parte, aparece información redundante (apéndices gramaticales muy simples, entradas independientes para adverbios de formación regular, diminutivos, ...). Cabe resaltar la pobreza de las instrucciones de uso y de manejo en muchos de ellos, que llegan al extremo de omitir la explicación de las abreviaturas gramaticales o las etiquetas, y que no se pueden encontrar en ningún otro sitio, en detrimento de información que se podría encontrar en otro tipo de obras, como gramáticas.

BIBLIOGRAFÍA

DICCIONARIOS

(a) diccionarios bilingües español-inglés:

CEI: Sмiтн, Colin (ed.) (1991): Collins Spanish-English English-Spanish Dictionary, Glasgow, HarperCollins.

LeI: CazalaÀ, B., P. Савот у A. J. РАLat (1993): Gran Diccionario Espa- 
ñol-inglés English Spanish, Paris, Larousse.

OSD: Styles, Carol, Stephanie Parker y Cristina Hülskamp (eds.) (1994): The Oxford Spanish Dictionary, New York, New York University Press.

(b) diccionarios bilingües español-francés:

LFE: García Pelayo y Gross, Ramón y Jean Testas (eds.) (1995): Grand Dictionnaire Espagnol-Français Français-Espagnol, Paris, Larousse.

SFE: MARTínez Amador, E. (ed.) (1984): Diccionario Francés-Español Español-Francés, Barcelona, Ramón Sopena.

(c) diccionarios bilingües español-italiano:

CDF: CARBOnElL, Sebastián (1994): Dizionario fraseologico completo Italiano Spagnolo Spagnolo Italiano, Milano, Ulrico Hoepli.

DST: TAM, Laura (1997): Dizionario Spagnolo-Italiano Italiano-Español, Milano, Ulrico Hoepli.

SEI: MARTínez AMADOR, E. (ed.) (1990): Diccionario Italiano-Español Español-Italiano, Barcelona, Ramón Sopena.

(d) diccionarios bilingües español-alemán:

SAE: MARTínez AMAdor, E. (ed.) (1991): Diccionario Alemán-Español Español-Alemán, Barcelona, Ramón Sopena.

DLEA: SlabÝ, Rudolf J. R. Grossman y C. Illig (1994): Diccionario de las lenguas española y alemana, Barcelona, Herder.

Klett (1997): Klett Vox Diccionario Avanzado Alemán. Español-Alemán Deutsch-Spanisch, Stuttgart, Klett.

(e) diccionarios bilingües español-portugués:

DPE: Martínez Almoyna, Julio (1998): Diccionário de Português-Espanhol Espanhol-Português, Porto, Porto Editora.

DEB: GARCíA, Hamílcar de (1998): Diccionário português espanhol espanhol português, São Paulo, Globo.

EB (2001): Gran Diccionario español-portugués português-espanhol, Madrid, Espasa.

REFERENCIAS BIBLIOGRÁFICAS

Ahumada, Ignacio (ed.) (1992): Diccionarios españoles. Contenido y aplicaciones, Jaén, Seminario de Lexicografía Hispánica.

AL-Kasimi, Alí (1977): Linguistics and bilingual dictionaries, Leiden, E. J. Brill. 
Alvar EzQuerra, M. (ed.) (1991): Euralex '90 Proceedings, Barcelona, Biblograf.

AtKINs, B. T. S. (ed.) (1998): Using dictionaries, Tubingen, Niemeyer.

DiAB, T. (1990): Pedagogical Lexicography, Tübingen, M. Niemeyer.

Gellerstam, M. et al. (eds.) (1996): Euralex '96 Proceedings I-II. Gotemburg, University/Department of Swedish.

HAENSCH, Günther et al. (1982): La lexicografia, Madrid, Gredos.

HAENSCH, Günther (1997): Los diccionarios del español en el umbral del siglo XXI: Problemas actuales de la lexicografia, Salamanca, Universidad de Salamanca.

Hartmann, Reinhardt R. K. (ed.) (1984): LEXeter '83 Proceedings, Tübingen, Niemeyer.

Hausmann, Franz Josef et al. (eds.) (1989-1991): Dictionaries. An international encyclopaedia of lexicography, Berlin, Walter de Gruyter.

HERnÁndez HeRnánDEZ, Humberto (ed.) (1994): Aspectos de lexicografía contemporánea, Barcelona, Biblograf.

Householder, Fred y Sol Saporta (eds.) (1967): Problems in lexicography, Bloomington, Indiana University Press.

HyldgaARD-Jensen, Karl y Arne ZetTersten (eds.) (1992): Symposium on Lexicography $V$, Tübingen, Niemeyer.

Landau, Sidney (1989): Dictionaries. The art and craft of lexicography, Cambridge, Cambridge University Press.

Magay, T. y J. Zigani (eds.) (1989): BudaLEX' '88 Proceedings, Budapest, Akademiai Kiado.

MARTínez De Sousa, José (1995): Diccionario de lexicografía práctica, Barcelona, Biblograf.

Piotrowski, Tadeusz (1994): Problems in Bilingual Lexicography, Wroclaw, Wydawnictwo Uniwersytetu Wroclawskiego.

SÁnchez Merino, Antonio (2002): Aspectos prácticos y teóricos de lexicografia bilingüe española, Tesis doctoral inédita.

SingH, Ram Adar (1982): An introduction to lexicography, Mysore, Central Institute of Indian Languages.

Svensen, Bo (1993): Practical lexicography, Oxford, Oxford University Press.

Tommola, H. et al. (eds.) (1992): EURALEX'92 Proceedings, Tampere, Yliopisto.

WotJAK, Gerd (ed.) (1992): Estudios de lexicografía y metalexicología del español actual, Tübingen, Niemeyer.

Zgusta, Ladislav (1971): Manual of lexicography, The Hague, Mouton. (1987): Lexicography today, Tübingen, Niemeyer. 